\title{
Maintenance pharmacotherapy of unipolar depression
}

\author{
Sam Forshall and David J. Nutt
}

\begin{abstract}
Aims and methods The purpose of this paper is to review current evidence and opinion with regard to the long-term treatment of unipolar depression. The method employed was a Psychlit search using the search items long-term, maintenance, treatment and depression.

Results The search yielded 91 articles.

Clinical implications Unipolar depression is frequently recurrent and sometimes a chronic illness. The paper identifies those at greatest risk of recurrence. It goes on to examine strategies to prevent relapse and for prophylactic treatment. It concludes that continuation treatment should be sustained at full dose for 4-6 months after full remission of symptoms. Where depression is highly recurrent the acute phase dose should be maintained in the long-term.
\end{abstract}

Major depression is both a common and serious psychiatric disorder. Epidemiological surveys indicate a one-year prevalence of $3 \%$, while the lifetime prevalence can be up to $20 \%$. Formerly, major depression was viewed as having a relatively good prognosis, however, long-term naturalistic community surveys have indicated that this is not the case as there is a high risk of recurrence and chronicity (Angst, 1992; Keller, 1994). Of those who have suffered one episode of depression $50-85 \%$ will have a subsequent episode, while those who have had two episodes 80-90\% will develop a third. With each recurrence a further recurrence becomes more likely and the period in remission tends to become shorter. Recurrent depression is costly, with each episode incurring not only the expense of treatment, but also a loss of productivity and disruption to social relationships, to say nothing of the distress to the sufferer. In addition. surveys consistently indicate that $15 \%$ of sufferers of depression go on to commit suicide. With each episode there is a considerable risk of long-term psychosocial impairment and chronicity, with estimates varying between $15-30 \%$. Thus, it becomes very important to develop strategies for the long-term management of depression.

\section{Definition of terms}

It is necessary to define terms, for within the literature there is considerable confusion with relapse and recurrence being used synonymously, while 'maintenance therapy', is used to mean either continuation or prophylactic treatment.

\section{Stages of treatment}

(a) Acute phase begins with first presentation. During this phase the goal of treatment is to elicit a treatment response.

(b) Continuation phase begins with a response to treatment. The treatment goals are to prevent a relapse and to convert the response to treatment into a remission.

(c) Prophylactic treatment or maintenance phase begins at the time the patient is deemed to have recovered from the episode of depression and in this phase the goal of treatment is to prevent the recurrence of another acute episode (American Psychiatric Association, 1993; Prien \& Kocsis, 1995).

\section{Outcomes}

(a) Response to treatment is a significant, but not total, reduction in symptoms following the initiation of treatment.

(b) Remission is the return of the patient to a level of functioning that is consistent with the patient's premorbid or well self. Often a Hamilton Rating Scale for Depression score (Hamilton, 1967) of less than seven or eight is used as the quantitative measure of remission.

(c) Recovery is a remission that has been sustained for such a time that the episode of depression is deemed to be over. normally 4-6 months.

(d) Relapse is a return of symptoms that meet the diagnostic criteria prior to the end of the index episode.

(e) Recurrence is a return of symptoms that meet the diagnostic criteria after a full recovery. 


\section{Continuation treatment}

The purpose of continuation treatment is to prevent a relapse. It is thought that antidepressant drugs can suppress the symptoms of the illness without correcting the underlying psychopathological process which continues its natural course. Withdrawal of medication during this time frequently results in the re-emergence of symptoms and relapse.

\section{Suitability for continuation treatment}

Everyone who has had an episode of depression which has remitted should be maintained on continuation therapy. However, certain groups are at particular risk of relapse. Factors associated with high risk of relapse include (Angst, 1992; Thase, 1992; Prien \& Kocsis, 1995):

(a) chronicity of depressive illness;

(b) severe index episode;

(c) presence of residual depressive symptoms:

(d) older age;

(e) poor social support:

(f) ongoing or renewed psychosocial stressors;

(g) history of previous non-affective psychiatric disorder;

(h) comorbid medical illness.

\section{Antidepressant dosage}

The need for continuation treatment should be discussed with the patient early in the acute phase of treatment. In general, it is appropriate to continue with both the medication and the full dosage that produced the acute phase response (Johnstone et al, 1990). Studies have shown that when the dosage is reduced rates of relapse increase (Frank et al, 1993). For this reason it is important to select an antidepressant that is well tolerated from the outset for, with prolonged treatment, side-effects can become increasingly irksome and lead to poor compliance and premature cessation of treatment.

\section{Duration of continuation treatment}

To prevent relapse pharmacological treatment should continue for about 4-6 months after an apparent full remission of symptoms (Frank et al, 1990: Montgomery, 1994). The persistence of even mild symptoms indicates that the patient is at high risk of relapse upon withdrawal. Discontinuation of treatment should be gradual ( $<25 \%$ of total dose per week) to prevent the problems of withdrawal phenomena (e.g. cholinergic syndrome with tricyclic antidepressants). During withdrawal, and after, the patient should continue to be monitored for re-emergence of depressive symptoms.

\section{Electroconvulsive therapy and continuation therapy}

During the continuation phase electroconvulsive therapy (ECT) has similar rates of relapse to antidepressants, and so ECT-induced remission also requires continuation therapy. Generally, this takes the form of antidepressant medication. In selecting an antidepressant, it must be remembered that research indicates that an antidepressant that was ineffective in treating the acute phase is likely to be equally so in the continuation phase. Where no effective antidepressant can be found, continuation of ECT should be considered (Sackeim et al, 1990).

\section{Prophylactic treatment}

The purpose of prophylactic treatment is to prevent the recurrence of major depression with the emergence of a new episode. Prophylaxis should be initiated in all who have suffered three or more depressive episodes, especially where there was a shorter period between them. It is also important to consider the likely impact that a further episode will have on the patient and their family. Furthermore, prophylaxis should be considered in all those who have had two episodes in two years (World Health Organization Mental Health Collaboration Centres, 1989). Risk factors for recurrence of depression include:

(a) previous recurrences especially if severe;

(b) early $(<25)$ and late $(>60)$ onset;

(c) chronicity;

(d) severe or life threatening index episode;

(e) seasonal pattern;

(f) family history of affective disorder;

(g) depression secondary to other psychiatric or medical diagnosis:

(h) presence of a sick spouse.

Factors which predict severity of a subsequent episode include:

(a) psychotic features (in previous episodes);

(b) serious suicide attempt (in previous episodes):

(c) serious functional impairment (in previous episodes):

(d) family history of suicide;

(e) family history of bipolar disorder, or psychotic affective illness.

In initiating prophylactic treatment it is important to involve the patient and their family in the decision-making process and to discuss with them the risks and benefits of both longterm treatment and the withdrawal of that treatment. The full support of both the patient and their family has been found to benefit longterm compliance. In addition, it is important to 
give consideration to ongoing psychosocial stressors and to arrange appropriate interventions.

\section{Choosing an antidepressant}

No treatment has been found to be a panacea, and in long-term trials treatment failure is found in about $25 \%$ of patients. While no particular antidepressant is found to be consistently better than others, there is evidence that some are less effective (e.g. nortriptyline in the elderly (Georgotis et al, 1989)). As a rule it is wise to continue with the treatment that was effective in the acute and continuation phases. The sideeffect profile and tolerability of medication must be given careful consideration from the outset of treatment, for they can greatly influence longterm compliance, particularly at a time when the patient may consider themselves 'cured'. Available evidence suggests that the selective seretonin reuptake inhibitors have demonstrable efficacy and are well tolerated (Montgomery, 1994).

The evidence for the role of lithium in maintenance therapy is equivocal. Meta-analysis of early research indicated its usefulness and subsequent, mainly European, studies have supported this view. Against this a major National Institute of Mental Health collaborative study found that maintenance treatment with lithium alone was little better than placebo while the combination of lithium with imipramine was no better than imipramine alone. Though there may have been methodological flaws in this study, its findings have been supported by a subsequent UK-based study comparing lithium with amitriptyline (Johnstone et al, 1990). Moreover lithium has significant disadvantages compared with antidepressants as it requires regular blood tests and has well established long-term side-effects. However, $10-15 \%$ of those people diagnosed as having a unipolar illness go on to develop bipolar affective disorder and where there is a strong suggestion of an as yet undiagnosed bipolar illness lithium may be preferable to antidepressants. Risk factors for an undiagnosed bipolar illness include a family history of bipolar affective disorder, early age of onset. psychomotor retardation and where there is a high frequency of depressive recurrence.

\section{Antidepressant dosage}

The evidence that exists, though not strong, suggests that prophylactic treatment should be maintained at the full dose effective in the acute phase (Montgomery, 1994). Under certain circumstances, for example, when side-effects are problematic, a reduction in dose may be justified in order to improve compliance.

\section{Duration of prophylactic treatment}

There are no valid predictors of when patients will no longer require preventative treatment (Prien \& Kocsis, 1995). Meta-analysis indicates the risk of recurrence diminishes after two years of sustained recovery, though even after three years the risk remains significant. As in initiating treatment, when considering its cessation it is necessary to discuss both the risks and benefits of alternative courses of action in a collaboration with the patient and their family. The stronger the indications for initiating prophylactic treatment the longer it should continue. In highly recurrent depression, successful treatment should be continued for life.

When it is decided to discontinue long-term treatment it should be withdrawn slowly over weeks or even months, and during this time the patient's mental state should be monitored closely for re-emergence of depressive symptoms.

\section{Special considerations}

\section{Psychotic depression}

Continuation therapy should employ a combination of antidepressant and antipsychotic medication and these should be maintained for six months (Thase, 1992). The dose of the neuroleptic should be the minimum required to sustain the remission. After six months of recovery the antipsychotic can be tapered off, unless the patient has suffered a recurrence on antidepressant medication alone, in which case it too should be continued.

\section{Recurrence during prophylaxis}

In trials $20-70 \%$ of patients have a recurrence despite prophylactic antidepressant treatment. When this occurs it is important to check the patients compliance with medication (Thase, 1992). In addition to this it is important to consider organic causes of depression, such as thyroid disease, the possibility of substance misuse as well as looking at psychosocial issues. After waiting to ensure that it is a recurrence rather than a brief 'flurry' of symptoms, options for management include the addition of lithium or switching to an alternative antidepressant.

\section{References}

American Psychlatric Assoclation (1993) Practice guidelines for major depressive disorder in adults. American Journal of Psychiatry. 160 (suppl.). 1-26.

ANGST, J. (1992) How recurrent and predictable is depressive illness? In Long-term Treatment of Depression (eds S. Montgomery \& F. Rouillon). New York: John Wiley. 
Frank. E., Kupfer, D., Pere, J., et al (1990) Three-year outcomes for maintenance therapies in recurrent depression. Archives of General Psychiatry. 47. 10931099.

- - - - - et al (1993) Comparison of full-dose versus halfdose pharmacotherapy in the maintenance treatment of recurrent depression. Journal of Affective Disorder, 27. $139-145$.

Georgotis, A., McCue, R. E. \& COOPER, T. B. (1989) A placebo-controlled comparison of nortriptyline and phenelzine in maintenance therapy of elderly depressed patients. Archives of General Psychiatry. 48. 783-786.

Hamilon, M. (1967) Development of a rating scale for primary depressive illness. British Journal of Social and Clinical Psychology, 6, 278-296.

Johnstone, W. C., OWENS, D. G. C., LAMBerT, M. T., et al (1990) Combination tricyclic antidepressant and lithium maintenance medication in unipolar and bipolar depressed patients. Journal of Affective Disorder, 20, 225-233.

KELLER, M. B. (1994) Depression: a long-term lliness. British Journal of Psychiatry. 166 (suppl. 26), 9-15.

MONTGOMERY, S. A. (1994) Long-term treatment of depression. British Joumal of Psychiatry. 165 (suppl. 26), 31-36.
PRIEN, R. F. \& Kocsis, J. H. (1995) Long-term treatment of mood disorders. In Psychopharmacology: the Fourth Generation of Progress (eds F. E. Bloom \& D. J. Kupfer), pp. 1067-1079. New York: Raven Press.

Sackeim, H., Prudic, J., Devanand, D. P., et al (1990) The impact of medication resistance and continuation pharmacotherapy on relapse following responses to electroconvulstve therapy in major depression. Journal of Clinical Psychopharmacology. 10, 96-104.

THASE, M. E. (1992) Long-term treatments of recurrent depressive disorders. Joumal of Clinical Psychiatry. 68 (suppl. 9), 32-44.

WORLD HEalth Organization MEnTal HEalth Collaborating CENTRES (1989) Pharmacotherapy of depressive disorders: a consensus statement. Journal of Affective Disorders, 17, 197-198.

*Sam Forshall, Senior Registrar; and David J. Nutt, Professor of Psychopharmacology and Head of Department of Clinical Medicine, Psychopharmacology Unit, School of Medical Sciences, University of Bristol BS8 1TD

*Correspondence

\section{Evidence-Base Briefing: Dementia Claire Palmer}

The number of published papers on the subject of dementia is constantly rising and it is virtually impossible for clinicians to read everything available, let alone to

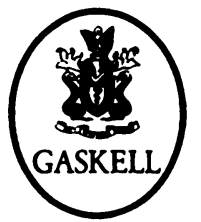
appraise it properly.

Evidence-Base Briefings (EBBs) are summarised collections of synthesised 'evidence' in a given topic area. This document on dementia attempts to encapsulate the best available evidence into a format which is quick and easy to use. Its main aim is to provide a checklist of appraised evidence from which a clinician can easily obtain original documents. These documents can then be appraised (using the tool provided) and interpreted for the clinician's own practice. The evidence sources on which the EBB is based include research, guidelines and national guidance. The EBB includes full references to its source documents and details of further information resources to support evidence-based practice.

June 1999, Paperback, ISBN 190124235 8, £15.00

Available from Book Sales, Royal College of Psychiatrists, 17 Belgrave Square, London SWIX 8PG Tel +44(0) 1712352351 (extension 146) $9.30 \mathrm{am}-2 \mathrm{pm}$, Fox +44 (0) 1712451231

http://mww.rcpsych.oc.uk 